Buli. Austral. Math. Soc.

VOL. 38 (1988) [231-238]

\title{
ALMOST PERIODIC SOLUTIONS OF NONLINEAR PARABOLIC EQUATIONS
}

YISONG YANG

\begin{abstract}
In this note the recent result of Corduneanu on almost periodicity of $L^{2}(G)$-bounded solutions of nonlinear parabolic equations is extended to the case when the nonlinear growth rate is beyond the first eigenvalue of the associated elliptic boundary value problem.
\end{abstract}

\section{INTRODUCTION}

Let $X$ be a Banach space. Recall that a continuous function $g: \mathbf{R} \rightarrow X$ is called $X$-almost periodic if for every $\varepsilon>0$ there is a relatively dense subset $T_{\varepsilon} \subset \mathbf{R}$ such that

$$
\sup _{t}\|g(t+\tau)-g(t)\|_{X}<\varepsilon, \forall \tau \in T_{\varepsilon} .
$$

Obviously the $X$-almost periodicity of $g: \mathbb{R} \rightarrow X$ implies the boundedness of the set $\{g(t): t \in \mathbb{R}\}$ in $X$.

In this note we are interested in obtaining $L^{2}(G)$-almost periodicity of $L^{2}(G)$ bounded solutions of the following semilinear parabolic equation

$$
\begin{aligned}
u_{t} & =\Delta u+f(t, x, u),(t, x) \in \mathbf{R} \times G, \\
u & =\mathbf{0} \text { on } \partial G
\end{aligned}
$$

where $G \subseteq \mathbf{R}^{n}$ is a bounded domain with sufficiently smooth boundary $\partial G$ and $f: \mathbf{R} \times$ $G \times \mathbf{R} \rightarrow \mathbf{R}$ satisfies the basic assumption (see $[1,2]$ ) in the following.

$\left(C_{0}\right) f(t, x, u)$ is continuous and $L^{2}(G)$-almost periodic in $t$, uniformly with respect to $u \in \mathbf{R}$.

One can arrange the eigenvalues of the associated elliptic problem

$$
\triangle u+\lambda u=0 \text { in } G, u=0 \text { on } \partial G
$$

in an ascending order $0<\lambda_{1}<\lambda_{2} \leqslant \lambda_{3} \leqslant \ldots$

The result of Corduneanu $[1,2]$ can be stated as follows.

\section{Received 18 November 1987}

Copyright Clearance Centre, Inc. Serial-fee code: 0004-9729/88 \$A2.00+0.00. 
THEOREM. Let $f_{u}: \mathbf{R} \times G \times \mathbf{R} \rightarrow \mathbf{R}$ be continuous and $u=u(t, x)$ be a $C^{2}(\bar{G})$ solution of the problem (1). If $u(t, x)$ is $L^{2}(G)$-bounded, that is, there is a constant $M>0$ such that

$$
\sup _{t} \int_{G} u^{2}(t, x) d x \leqslant M
$$

then $u(t, x)$ is $L^{2}(G)$-almost periodic (if $f(t, x, u)$ is periodic in $t$ instead, then so is $u(t, x))$ provided $f_{u} \leqslant \mu<\lambda_{1}$ in $\mathbf{R} \times G \times \mathbf{R}$.

IIis proof is based on the Poincaré inequaltiy. In this note the $L^{2}(G)$-almost periodicity will be established assuming

$$
\text { closure }\left(f_{u}(\mathbf{R} \times G \times \mathbf{R})\right) \cap\left\{\lambda_{j}: j=1,2, \ldots\right\}=\emptyset .
$$

Our argument below is based on an eigenfunction expansion. The extension of this method to some more general problems is immediate (see Section 3).

Note that conditions of type $\left(C_{1}\right)$ occur in various other problems such as the unique solvability of nonlinear integral equations [3], the existence of solutions of boundary value problems $[4,5]$, and also the existence of periodic solutions of parabolic equations $[4,6]$.

\section{MaIN Result}

Suppose $f, f_{u}: \mathbf{R} \times G \times \mathbf{R} \rightarrow \mathbf{R}$ are both continuous and satisfy $\left(C_{0}\right)$ and $\left(C_{1}\right)$ (where we assume $G$ is connected to simplify the statement of the result).

TheOREM 1. Let $u=u(t, x)$ be a $C^{2}(\bar{G})$ solution of the problem (1). If $u(t, x)$ is $L^{2}(G)$-bounded, then $u(t, x)$ is $L^{2}(G)$-almost periodic (if $f(t, x, u)$ is periodic in $t$ instead, then so is $u(t, x))$.

Proof: First consider the problem

$$
\begin{aligned}
u_{t}-(\Delta u+m u) & =v(t, x),(t, x) \in \mathbf{R} \times G, \\
u & =0 \text { on } \partial G .
\end{aligned}
$$

Suppose $u$ and $v$ are both $L^{2}(G)$-bounded and

$$
\lambda_{j}<m<\lambda_{j+1} \text { for some integer } j \geqslant 1 \text {. }
$$

We claim that

$$
\sup _{t} \int_{G} u^{2}(t, x) d x \leqslant \max \left(\left[m-\lambda_{j}\right]^{-2},\left[m-\lambda_{j+1}\right]^{-2}\right) \sup _{t} \int_{G} v^{2}(t, x) d x .
$$


Let $u_{k}(x)$ be the eigenfunction of the problem (2) associated with the $k$ th eigenvalue $\lambda_{k}$. Suppose $\left\{u_{k}\right\}$ is an orthonormal basis of $L^{2}(G)$. We have, in the space $L^{2}(G)$,

$$
u(t, x)=\sum a_{k}(t) u_{k}(x), v(t, x)=\sum b_{k}(t) u_{k}(x)
$$

Hence we have, by the Parseval equality and the $L^{2}(G)$-boundedness of $u$ and $v$, the inequalities

$$
\begin{aligned}
& \sum a_{k}^{2}(t)=\int_{G} u^{2}(t, x) d x \leqslant M \\
& \sum b_{k}^{2}(t)=\int_{G} v^{2}(t, x) d x \leqslant M
\end{aligned}
$$

where $M>0$ is a constant independent of $t \in \mathbf{R}$. It follows from inserting (6) into (3) that

$$
\dot{a}_{k}(t)+\left(\lambda_{k}-m\right) a_{k}(t)=b_{k}(t), t \in \mathbf{R}, k=1,2, \ldots
$$

Consequently for any given $T \in \mathbf{R}$,

$$
a_{k}(t)=a_{k}(T) e^{\left(\lambda_{k}-m\right)(T-t)}+\int_{T}^{t} b_{k}(s) e^{\left(\lambda_{k}-m\right)(s-t)} d s .
$$

If $k \leqslant j$, from (10) we have

$$
\begin{aligned}
\left|a_{k}(t)\right| \leqslant & \left|a_{k}(T)\right| e^{\left(\lambda_{k}-m\right)(T-t)} \\
& +\left(m-\lambda_{k}\right)^{-1}\left(1-e^{\left(\lambda_{k}-m\right)(T-t)}\right) \sup \left|b_{k}(s)\right|
\end{aligned}
$$

for $T>t$. From the boundedness of $a_{k}(t)$ and $b_{k}(t)$ (see (7), (8)) and $\lambda_{k}<m$ and taking $T \rightarrow \infty$ in (11) we obtain

$$
\sup _{t}\left|a_{k}(t)\right| \leqslant\left(m-\lambda_{k}\right)^{-1} \sup _{t}\left|b_{k}(t)\right|, k=1,2, \ldots, j .
$$

Similarly from (10) one can establish the inequality

$$
\sup _{t}\left|a_{k}(t)\right| \leqslant\left(\lambda_{k}-m\right)^{-1} \sup \left|b_{k}(t)\right|, k=j+1, j+2, \ldots
$$

(5) is now an immediate implication of (12) and (13).

Let $u=u(t, x)$ be a solution of (1) and satisfy the assumption of the theorem. 
For given $\tau \in \mathbf{R}$ define $v=w(t, x)=u(t+\tau, x)-u(t, x)$. Then $w$ satisfies the equation

$$
\begin{aligned}
w_{t}-\triangle w & =f_{u}(t+\tau, x, U(t, x)) w+f(t+\tau, x, u(t, x))-f(t, x, u(t, x)) \text { in } \mathbf{R} \times G \\
w & =\mathbf{0} \text { on } \partial G
\end{aligned}
$$

where $U(t, x)$ is between $u(t, x)$ and $u(t+\tau, x)$. The condition $\left(C_{1}\right)$ is equivalent to the following statements.

$\left(C_{1}\right)_{a}$ There is a constant $\mu$ such that $f_{u} \leqslant \mu<\lambda_{1}$ in $\mathbf{R} \times G \times \mathbf{R}$, or otherwise

$\left(C_{1}\right)_{b}$ There are constants $p, q$ and integer $j \geqslant 1$ such that

$$
\lambda_{j}<p \leqslant f_{u} \leqslant q<\lambda_{j+1} \text { for }(t, x, u) \in \mathbf{R} \times G \times \mathbf{R} .
$$

$\left(\left(C_{1}\right)_{a}\right.$ is the condition imposed in Corduneanu $\left.[1,2]\right)$. We must now prove the theorem under condition $\left(C_{1}\right)_{b}$.

Rewrite (14) as

$$
\begin{aligned}
w_{t}-(\Delta w+m w) & =\left(f_{u}(t+\tau, x, U)-m\right) w+f(t+\tau, x, u(t, x)) \\
& -f(t, x, u(t, x)) \text { in } \mathbf{R} \times G \\
w & =0 \text { on } \partial G
\end{aligned}
$$

where $p<m<q$. Applying the estimate (5) to the equation (15) we arrive immediately at the inequality

$$
\begin{aligned}
& \sup _{t}\left(\int_{G} w^{2}(t, x) d x\right)^{\frac{1}{2}} \leqslant c^{\frac{1}{2}}(m) \sup _{t}\left(\int_{G} w^{2}(t, x) d x\right)^{\frac{1}{2}} \\
& \quad+d^{\frac{1}{2}}(m) \sup _{t}\left(\int_{G}[f(t+\tau, x, u(t, x))-f(t, x, u(t, x))]^{2} d x\right)^{\frac{1}{2}}
\end{aligned}
$$

where $c(m) \equiv \max \left(\left[m-\lambda_{j}\right]^{-2},\left[m-\lambda_{j+1}\right]^{-2}\right) \max \left([m-p]^{2},[m-q]^{2}\right)$ and $d(m)=$ $\max \left(\left[m-\lambda_{j}\right]^{-2},\left[m-\lambda_{j+1}\right]^{-2}\right)$. Obviously we can choose $m$ to make $c(m)<1$. It then follows from (16) that

$$
\begin{gathered}
\sup _{t}\left(\int_{G}[u(t+\tau, x)-u(t, x)]^{2} d x\right)^{\frac{1}{2}} \\
\leqslant\left[d^{\frac{1}{2}}(m) /\left(1-c^{\frac{1}{2}}(m)\right)\right] \sup _{t}\left(\int_{G}[f(t+\tau, x, u(t, x))-f(t, x, u(t, x))]^{2} d x\right)^{\frac{1}{2}} .
\end{gathered}
$$

The proof of the theorem is complete. 


\section{A MORE GENERAL THEOREM}

The method in Section 2 can be applied to some more general problems concerning almost periodicity.

Let $G$ be a bounded connected domain in $\mathrm{R}^{n}$. Let $D_{j}=\partial / \partial x_{j}, D=$ $\left(D_{1}, \ldots, D_{n}\right), D^{\alpha}=D_{1}^{\alpha_{1}} \ldots D_{n}^{\alpha_{n}}, \alpha=\left(\alpha_{1}, \ldots, \alpha_{n}\right)$, and $|\alpha|=\alpha_{1}+\ldots+\alpha_{n}$. Let

$$
P(x, D)=\sum_{|\alpha| \leqslant 2 m} A_{\alpha}(x) D^{\alpha},
$$

be an elliptic operator on $G$ of order $2 m$ with the principal part

$$
p(x, D)=\sum_{|\alpha|=2 m} A_{\alpha}(x) D^{\alpha} .
$$

$m$ boundary operators $\left\{Q_{j}: j=1, \ldots, m\right\}$ of respective orders $m_{j}<2 m$ are defined by

$$
Q_{j}(x, D)=\sum_{|\alpha| \leqslant m_{j}} B_{j \alpha}(x) D^{\alpha}, j=1, \ldots, m,
$$

with the principal parts

$$
q_{j}(x, D)=\sum_{|\alpha|=n_{j}} B_{j \alpha}(x) D^{\alpha}, j=1, \ldots, m .
$$

The following standard regularity conditions are assumed:

(i) $\partial G \in C^{2 m}, A_{\alpha} \in C^{|\alpha|}(\bar{G}), B_{j \alpha} \in C^{2 m-m_{j}}(\partial G)$, and $m_{i} \neq m_{j}$ if $i \neq j$;

(ii) at any point $x \in \partial G$ let $\vec{n}$ be an outward normal to $\partial G$ at $x$ and $\vec{t}$ a nonzero tangent vector of $\partial G$ at $x$. Then the polynomials $q_{j}(x, \vec{t}+z \vec{n})$ in $z$ are linearly independent modulo the polynomial $\left(z-z_{1}(\vec{t})\right) \ldots\left(z-z_{m}(\vec{t})\right)$ where $z_{j}(\vec{t})$ are the roots of $p(x, \vec{t}+z \vec{n})$ (as a polynomial in $z$ ) with positive imaginary parts. As a polynomial in $z, \operatorname{deg}\left(q_{j}\right)=m_{j}$ for any fixed $x \in \partial G$;

(iii) the coefficients of $P$ and $Q_{j}$ are all real.

Let $H^{2 m}\left(G ;\left\{Q_{j}\right\}\right)$ be the closure in $H^{2 m}(G)=W^{2 m, 2}(G)$ of the set of functions $u$ in $C^{2 m}(\bar{G})$ that satisfy the boundary conditions

$$
Q_{j} u=0 \text { on } \partial G, j=1, \ldots, m .
$$

Then $P u=P(x, D) u$ can be defined as an operator $P: L^{2}(G) \rightarrow L^{2}(G)$ with $\operatorname{Dom}(P)=H^{2 m}\left(G ;\left\{Q_{j}\right\}\right)$. Consider the evolution equation in the space $H^{2 m}\left(G ;\left\{Q_{j}\right\}\right)$

$$
u_{t}=P u-f(t, x, u),(t, x) \in \mathbf{R} \times G,
$$

where $f$ satifies the basic assumption $\left(C_{0}\right)$. 
TheOREM 2. Suppose $u=u(t, x)$ is a solution to the equation (17) in the sense that $u \in C^{1}\left(\mathbf{P}, H^{2 m}\left(G ;\left\{Q_{j}\right\}\right)\right)$. If $P$ is self-adjoint and $\operatorname{Ker}(P-c)=\{0\}$ for some $c \in \mathbb{P}$, then $u$ is $L^{2}(G)$-almost periodic provided $u$ is $L^{2}(G)$-bounded and

$$
\operatorname{closure}\left(f_{u}(\mathbf{R} \times G \times \mathbf{R})\right) \cap \sigma(P)=\emptyset
$$

where $\sigma(P)$ is the spectrum of $P$ and $f_{u}: \mathbf{P} \times G \times \mathbf{R} \rightarrow \mathbf{R}$ is continuous.

Proof: It is well-known that the assumptions on $P$ ensure that $\sigma(P)$ consists of all eigenvalues of $P$ which is an isolated countable subset of $R$, say, $\ldots \leqslant \lambda_{j} \leqslant \lambda_{j+1} \leqslant$ $\ldots$, and the corresponding set of eigenfunctions $\left\{u_{j}\right\}$ of $P$ can be chosen to be an orthonormal basis of $L^{2}(G)$.

We can conclude from (18) and the connectedness of closure $\left(f_{u}(\mathbf{R} \times G \times \mathbf{A})\right)$ that one of the following three situations must happen:

(a) there is an integer $k$ such that $\lambda_{k}<\lambda_{k+1}$ and

$$
\lambda_{k}<p \equiv \inf _{D} f_{u}(t, x, u) \leqslant \sup _{D} f_{u}(t, x, u) \equiv q<\lambda_{k+1}
$$

(b) $d_{1} \equiv \sup _{D} f_{u}(t, x, u)<\inf \sigma(P)$;

(c) $d_{2} \equiv \inf _{D} f_{u}(t, x, u)>\sup \sigma(P)$;

where $D \equiv \mathbf{R} \times G \times \mathbf{R}$, and $d_{1}$ and $d_{2}$ lie in $\mathbf{R}$.

The proof for (a) is the same as that for Theorem 1. The proofs for (b) and (c) are similar. Let us treat (c) first.

One can rearrange the eigenvalues of $P$ in a descending order

$$
\ldots \leqslant \lambda_{2} \leqslant \lambda_{1}<d_{2} \text {. }
$$

We still denote by $\left\{u_{j}\right\}$ the corresponding set of eigenfunctions which forms an orthonormal basis of $L^{2}(G)$. Put $w=w(t, x)=u(t+\tau, x)-u(t, x)$. We have

$$
w_{t}=P w-f_{u}(t+\tau, x, U(t, x)) w-[f(t+\tau, x, u(t, x))-f(t, x, u(t, x))] .
$$

It follows easily from the eigenfunction expansion $w(t, x)=\sum c_{j}(t) u_{j}(x)$ that

$$
\left.(P w, w)_{2} \leqslant \lambda_{1}(w, w)_{2} \quad \text { (where }(., .)_{2} \text { is the inner product of } L^{2}(G)\right) \text {. }
$$

In virtue of $(19),(20),(21)$, and the Schwarz inequality we obtain

$$
\begin{aligned}
\frac{d}{d t}\left[\frac{1}{2}(w, w)_{2}\right] & \leqslant\left(\lambda_{1}-d_{2}\right)(w, w)_{2} \\
& +(w, w)_{2}^{\frac{1}{2}}\left(\int_{G}[f(t+\tau, x, u(t, x))-f(t, x, u(t, x))]^{2} d x\right)^{\frac{1}{2}}
\end{aligned}
$$


From (22) the conclusion of the theorem follows immediately (see [1]).

In case (b) one can rearrange the eigenvalues of $P$ in an ascending order

$$
d_{1}<\lambda_{1} \leqslant \lambda_{2} \leqslant \ldots
$$

An eigenfunction expansion gives us $(P \cdot w, w)_{2} \geqslant \lambda_{1}(w, w)_{2}$. Therefore (22) is replaced by

$$
\frac{1}{2} \dot{a}(t) \geqslant\left(\dot{\lambda}_{1}-d_{1}\right) a(t)-a^{\frac{1}{2}}(t) b^{\frac{1}{2}}(t), t \in \mathbf{R},
$$

where $a(t) \equiv(w, w)_{2}$ and

$$
b(t) \equiv \int_{G}[f(t+\tau, x, u(t, x, u(t, x)))-f(t, x, u(t, x))]^{2} d x .
$$

Choose $T>t$. This yields, from (23), that

$$
a(t) \leqslant a(T) e^{-2 c(T-t)}+\int_{t}^{T} 2 a^{\frac{1}{2}}(s) b^{\frac{1}{2}}(s) e^{-2 c(s-t)} d s
$$

where $c \equiv \lambda_{1}-d_{1}$. Put $M_{a}=\sup _{t} a(t)$ and $M_{b}=\sup _{t} b(t)$. Then (24) gives us the estimate

$$
a(t) \leqslant M_{a} e^{-2(T-t)}+M_{a}^{\frac{1}{2}} M_{b}^{\frac{1}{2}}\left(1-e^{-2 c(T-t)}\right) / c .
$$

Letting $T \rightarrow \infty$ in (25) we reach $M_{a} \leqslant M_{b} / c^{2}$. The $L^{2}(G)$-almost periodicity of $u(t, x)$ again follows.

Note. In Theorem 2 , if $f$ is periodic in $t$ then so is $u$.

Example. Consider the equation

$$
u_{t}=\triangle^{3} u+f(t, x, u) \text { in } \mathrm{R} \times G, \quad u=\partial u / \partial n=\triangle^{2} u=0 \text { on } \partial G
$$

in the space $H^{6}\left(G ;\left\{I d, \partial / \partial n, \triangle^{2}\right\}\right)$. It can be checked that (26) satisfies the assumption of Theorem 2, and the eigenvalues of the associated elliptic boundary value problem

$$
\triangle^{3} u=\lambda u \text { in } G, u=\partial u / \partial n=\triangle^{2} u=0 \text { on } \partial G,
$$

lie in the open interval $\mathbf{R}_{-}=(-\infty, 0)$. Suppose $f$ satisfies the condition $\left(C_{0}\right)$ and (26) has a solution $u \in C^{1}\left(\mathbf{R}, H^{6}\left(G ;\left\{I d, \partial / \partial n, \triangle^{2}\right\}\right)\right)$. Then the $L^{2}(G)$-boundedness of $u$ implies its $L^{2}(G)$-almost periodicity provided $f_{u} \leqslant 0$ for instance. 
Note. Under the assumptions of Theorem 1 or 2 the equation (1) or (17) has at most one $L^{2}(G)$-bounded solution.

For example, assume that $u, v$ are two $L^{2}(G)$-bounded solutions of Equation (17) in the sense that $u, v \in C^{1}\left(\mathbf{R}, H^{2 m}\left(G\left\{Q_{j}\right\}\right)\right)$ and $f$ satisfies condition (a) in the proof of Theorem 2. Then $w=u-v$ satisfies the equation

$$
w_{t}=P w-f_{u}(t, x, U(t, x)) w
$$

where $U(t, x)$ is between $u(t, x)$ and $v(t, x)$.

Let $m \in(p, q)$. As in the proof of Theorem 1, an eignefunction expansion technique proves the validity of an analogous version of the inequality (5) for the equation

$$
w_{t}-(P-m) w=W(t, x)
$$

where $w$ and $W$ are $L^{2}(G)$-bounded. From that one derives the estimate

$$
\sup _{t} \int_{G} w^{2}(t, x) d x \leqslant c(m) \sup _{t} \int_{G} w^{2}(t, x) d x
$$

where $c(m)=\max \left(\left[m-\lambda_{k}\right]^{-2},\left[m-\lambda_{k+1}\right]^{-2}\right) \max \left([m-p]^{2},[m-q]^{2}\right)$. When $m$ is suitably chosen so that $c(m)<1$, one must have $w=0$.

\section{REFERENCES}

[1] C. Corduneanu, 'Almost periodic solutions to nonlinear elliptic and parabolic equations', TMA, Nonlinear Anal. 7 (1983), 357-363.

[2] C. Corduneanu, 'Almost periodic solutions to some nonlinear parabolic equations', in Trends in Theory and Practice of Nonlinear Differential Equations, (ed. V. Lakshmikantham), pp. 139-141. (Marcel Dekker, Inc., New York and Basel).

[3] C. L. Dolph, 'Nonlinear integral equations of the Hammerstein type', Trans. Amer. Math. Soc. B6 (1949), 289-307.

[4] S. Fucik, Solvability of Nonlinear Equations and Boundary Value Problems (Reidel, Dordrecht, Boston, London, 1980).

[5] A. C. Lazer and D. E. Leach, 'On a nonlinear two point boundary value problem', J. Math. Anal. Appl. 20 (1969), 20-27.

[6] O. Vejvoda, Partial Differential Equations: Time-Periodic Solutions (Martinus Nijhoff, Boston and London, 1982).

Department of Mathematics and Statistics

Univesity of Massachusetts

Amlierst, MA 01003

United States of America 